\title{
Addition of Endothelin A-Receptor Blockade Spoils the Beneficial Effect of Combined Renin-Angiotensin and Soluble Epoxide Hydrolase Inhibition: Studies on the Course of Chronic Kidney Disease in 5/6 Nephrectomized Ren-2 Transgenic Hypertensive Rats
}

\author{
Věra Čertíková Chábováa, b Petr Kujal ${ }^{b, c}$ Zdeňka Vaňourkováb \\ Petra Škaroupkováb Janusz Sadowskid Elzbieta Kompanowska-Jezierskad \\ Vladimír Tesařa Bruce Hammock ${ }^{e}$ John Imig ${ }^{f}$ Hana Maxováb, g \\ Luděk Červenkab,g Ivana Vaněčkováh \\ aDepartment of Nephrology, 1st Faculty of Medicine, Charles University, Prague, Czech \\ Republic; ${ }^{b}$ Center for Experimental Medicine, Institute for Clinical and Experimental \\ Medicine, Prague, Czech Republic; ${ }^{C}$ Department of Pathology, 3rd Faculty of Medicine, \\ Charles University, Prague, Czech Republic; ${ }^{\mathrm{d}}$ Department of Renal and Body Fluid \\ Physiology, Mossakowski Medical Research Centre, Polish Academy of Sciences, Warsaw, \\ Poland; e Department of Entomology and UCD Cancer Center, University of California, Davis, \\ CA, USA; fDepartment of Pharmacology and Toxicology, Medical College of Wisconsin, \\ Milwaukee, WI, USA; ${ }^{9}$ Department of Pathophysiology, 2nd Faculty of Medicine, Charles

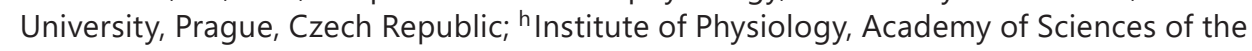 \\ Czech Republic, Prague, Czech Republic
}

\section{Keywords}

Chronic kidney disease · 5/6 Renal mass reduction - Hypertension · Soluble epoxide hydrolase inhibitor · Renin-angiotensin system - Endothelin A receptor blocker

\begin{abstract}
Introduction: Previous studies in Ren-2 transgenic hypertensive rats (TGR) after 5/6 renal ablation (5/6 NX) have shown that besides pharmacological blockade of the renin-angiotensin system (RAS) also increasing kidney tissue epoxyeicosatrienoic acids (EET) levels by blocking soluble epoxide hydrolase (sEH), an enzyme responsible for degradation of EETs, and endothelin type $A\left(E T_{A}\right)$ receptor blockade retards chronic kidney disease (CKD) progression. This
\end{abstract}




\section{Kidney \\ Blood Pressure \\ Research}

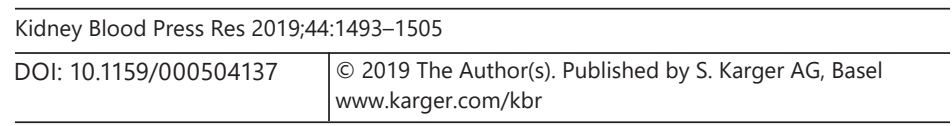

Čertíková Chábová et al.: RAS, sEH, and $\mathrm{ET}_{\mathrm{A}}$ Blockade in 5/6 Renal Ablation

prompted us to evaluate if this progression will be alleviated by the addition of $s E H$ inhibitor and $\mathrm{ET}_{\mathrm{A}}$ receptor antagonist to the standard complex blockade of RAS (angiotensin-converting enzyme inhibitor plus angiotensin II type 1 receptor blocker) in rats with established CKD. Methods: The treatment regimens were initiated 6 weeks after 5/6 NX in TGR, and the followup period was 60 weeks. Results: The addition of sEH inhibition to RAS blockade improved survival rate, further reduced albuminuria and renal glomerular and kidney tubulointerstitial injury, and attenuated the decline in creatinine clearance - all this as compared with 5/6 NX TGR treated with RAS blockade alone. Addition of $\mathrm{ET}_{\mathrm{A}}$ receptor antagonist to the combined RAS and sEH blockade not only offered no additional renoprotection but, surprisingly, also abolished the beneficial effects of adding SEH inhibitor to the RAS blockade. Conclusion: These data indicate that pharmacological strategies that combine the blockade of RAS and sEH could be a novel tool to combat the progression of CKD. Any attempts to further extend this therapeutic regimen should be made with extreme caution.

(C) 2019 The Author(s)

Published by S. Karger AG, Basel

\section{Introduction}

Chronic kidney disease (CKD) has become a growing public health problem [1, 2]. The natural course of progression from CKD to end-stage renal disease (ESRD) is known to be independent of the initial cause. However, the mechanisms underlying this appear to be quite similar [3-5]. It is generally agreed that diabetes mellitus, hypertension, and increased intrarenal activity of the renin-angiotensin system (RAS) are the most important factors influencing the progression of CKD to ESRD [4-8]. Therefore, the current "gold renoprotection paradigm" in patients with CKD is the antihypertensive therapy based on the RAS blockade, which has been shown to slow the progression of CKD to ESRD and attenuate cardiovascular disease [5, 8, 9]. However, the effectiveness of RAS blockade is limited, especially in advanced CKD. Apparently, new therapeutic regimens targeting hypertension in CKD are needed; however, no new drug for the treatment of CKD has been registered since 2001. This is so even though experimental studies suggested potential targets for novel treatments with substantial pertinent efforts in clinical research [10-12]. In order to study pathophysiological mechanism(s) of CKD and possible novel therapeutic measures to slow the progression of CKD to ESRD, the 5/6 renal ablation model is used [4, 13-16].

In the search for new pharmacological strategies targeting systems other than the RAS, attention has been recently directed to the endothelin (ET) system and to epoxyeicosatrienoic acids (EETs), cytochrome P-450 (CYP)-dependent metabolites of arachidonic acid.

ET-1 plays an important role in the pathophysiology of hypertension and end-organ damage, owing to the stimulation of type A receptors for ET-1 (endothelin type A [ET ${ }_{\mathrm{A}}$ ]) [17$22]$. The ET system is also activated in 5/6 NX model, and pharmacological blockade of either $\mathrm{ET}_{\mathrm{A}}$ alone or combined with complex pharmacological RAS inhibition was reported to be renoprotective in 5/6 NX Ren-2 renin transgenic rats (TGRs) - a model of angiotensin II-dependent hypertension with marked endogenous RAS activation [18, 19, 22-24].

EETs exhibit antihypertensive actions that are related both to their vasodilatory properties and to inhibition of the renal tubular sodium transport. Intrarenal EETs might operate as a compensatory system to counterbalance inappropriately increased RAS activity $[25,26]$. In addition, increasing renal EETs availability by blocking soluble epoxide hydrolase (sEH), an enzyme that converts EETs to biologically inactive dihydroxyeicosatrienoic acids, either alone or combined with standard RAS blockade, was reported to provide renoprotection in $5 / 6$ NX TGR $[27,28]$. 


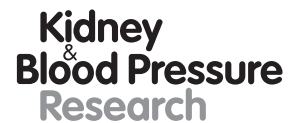

Research

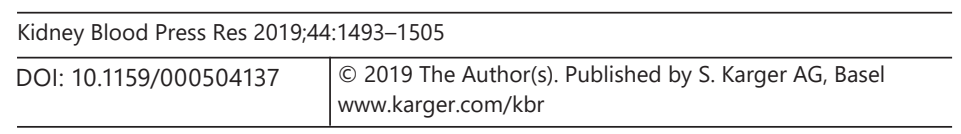

Čertíková Chábová et al.: RAS, sEH, and $\mathrm{ET}_{\mathrm{A}}$ Blockade in 5/6 Renal Ablation

Nevertheless, it has to be admitted that only a small minority of the mentioned experimental results could be translated into clinical practice. The common limitation is that treatment was initiated almost immediately after $5 / 6 \mathrm{NX}[3,4,15,16,22,29]$. Moreover, it has not been established if addition of $\mathrm{ET}_{\mathrm{A}}$ receptor blockade to the standard RAS blockade alone, or to the combined inhibition of sEH and RAS, would result in additive renoprotective effects.

Therefore, in the present study, we hypothetized whether the renoprotective effectiveness of combined RAS, $\mathrm{sEH}$, and $\mathrm{ET}_{\mathrm{A}}$ receptor blockade (as compared with those of RAS blockade alone) in 5/6 NX TGR would be efficient even in the animals with already established CKD, explicitly, in TGR that were left untreated until 6 weeks after 5/6 NX ("late treatment protocol") $[28,30]$. This model combines two critically important detrimental features promoting progression of CKD to ESRD: hypertension and increased intrarenal RAS activity $[19,30,31]$.

\section{Methods}

Animals

Heterozygous TGRs were used and age-matched Hannover-Sprague Dawley (HanSD) rats served as transgene-negative normotensive controls [23].

\section{Pharmacological Therapeutic Regimens}

We and others have demonstrated that the pharmacological blockade using combination of ACEi and $\mathrm{AT}_{1}$ blocker at increased doses provides a better cardio- and renoprotection [30$32]$. Thus, similarly as in our recent studies [28, 30], for the RAS blockade, a combination of trandolapril (6 mg/L drinking water, Gopten; Abbot, Prague, Czech Republic) and of losartan (Lozap, 100 mg/L drinking water, Zentiva, Prague, Czech Republic) was used.

The pharmacological blockade of sEH was achieved using the sEH inhibitor, cis-4-(4-[3adamantan-1-yl-ureido] cyclohexyloxy) benzoic acid, which was prepared freshly and given in drinking water at $3 \mathrm{mg} / \mathrm{L}[27,33]$.

$\mathrm{ET}_{\mathrm{A}}$ receptor blockade was achieved with atrasentan $\left(5 \mathrm{mg} \mathrm{kg}^{-1} \mathrm{day}^{-1}\right.$ in drinking water; Abbott, IL, USA). The dose of atrasentan was adjusted weekly to actual water intake; such dosage was previously found to effectively block $\mathrm{ET}_{\mathrm{A}}$ receptors $[30,33,34]$.

\section{Evaluation of Indices of Kidney Injury and of Creatinine Clearance}

At the end of the appropriate follow-up period, animals were killed, and the kidneys were used to assess renal glomerular damage and tubulointerstitial injury. The kidneys were fixed in $4 \%$ formaldehyde and embedded in paraffin. Sections were stained with hematoxylin-eosin and periodic acid Schiff reaction then examined and evaluated in a blind-test fashion. Fifty glomeruli in each kidney were examined for glomerulosclerosis index (GSI) on a semi-quantitative scale as described previously [28, 30]. Renal cortical tubulointerstitial injury was evaluated as defined by Nakano et al. [35] and as used in our studies $[28,30,31]$.

Urinary rat albumin was measured by a quantitative sandwich enzyme immunoassay technique, using the commercially available ELISA kit (ERA3201-1, AssayPro, St. Charles, MO, USA). Plasma creatinine was measured by FUJI DRI-CHEM analyzer using appropriate slides for creatinine CRE-P III (FUJIFILM Corp., Tokyo, Japan). Urine creatinine was determined using Liquick Cor-CREATININE kit (PZ CORMAY S.A., Poland), measured at $500 \mathrm{~nm}$. Clearance of creatinine was calculated using standard formula and was normalized per body weight. 


\section{Detailed Experimental Design}

Series 1: The Effects of Treatments on Survival Rate, Albuminuria, and Clearance of Endogenous Creatinine

In the week labeled -6 , at the age of 6 weeks, male HanSD rats and TGR from several litters were exposed to 5/6 NX under general anesthesia (tiletamine + zolazepam, Virbac SA, Carros Cedex, France, 8 mg/kg; and xylasine, Spofa, Czech Republic, 4 mg/kg intramuscularly) as described previously [28, 30, 31]. After $24 \mathrm{~h}$ recovery, the animals were randomly assigned to experimental groups and were left with no treatment. Six weeks later (in the week 0 ), an appropriate treatment regimen was initiated or rats were left without treatment. The following experimental groups were investigated:

1 Sham-operated HanSD rats + water (initial $n=10$ )

2 Sham-operated TGR + water $(n=11)$

$35 / 6 \mathrm{NX}$ TGR + water $(n=24)$

$45 / 6$ NX TGR + RAS blockade $(n=27)$

5 5/6 NX TGR + RAS blockade + sEH blockade $(n=28)$

$65 / 6 \mathrm{NX}$ TGR + RAS blockade + sEH blockade $+\operatorname{ET}_{\mathrm{A}}$ blockade $(n=31)$.

The follow-up period was until week +60 . In the weeks labeled $0,2,6,10,14,24,40$, and 50 and after appropriate habituation training, the animals were placed in individual metabolic cages, and their $24 \mathrm{~h}$ urine was collected for determination of daily albuminuria and urinary creatinine excretion.

Series 2: The Effects of Treatments on the Development of Renal Glomerular Damage and Renal Cortical Tubulointerstitial Injury

Animals were again exposed to the same procedure (i.e., 5/6 NX or sham operation) as in series 1 . In the week 0 ( 6 weeks after $5 / 6 \mathrm{NX}$ or sham operation), the following groups were studied:

1 Sham-operated HanSD rats + water $(n=6)$

2 Sham-operated TGR + water $(n=7)$

$35 / 6 \mathrm{NX}$ TGR + water $(n=12)$.

In the weeks labeled 2,6 , and 24 , the following separate groups were investigated ( $n$ represents always the number of animals at the time point of examination):

1 Sham-operated HanSD rats + water $(n=6)$

2 Sham-operated TGR + water $(n=7)$

$35 / 6 \mathrm{NX}$ TGR + water $(n=9)$

$45 / 6$ NX TGR + RAS blockade $(n=11)$

$5 \quad 5 / 6$ NX TGR + RAS blockade + sEH blockade $(n=10)$

$65 / 6$ NX TGR + RAS blockade + sEH blockade + ETA blockade $(n=11)$.

At the end of the appropriate follow-up period, animals were killed, and renal glomerular damage and kidney tubulointerstial injury were assessed. The aim was (a) to evaluate the course of renal damage in untreated and treated animals with established CKD throughout the critical periods of the experiment; (b) to investigate whether the addition of sEH inhibitor and $\mathrm{ET}_{\mathrm{A}}$ receptor antagonist to standard RAS blockade will exhibit some beneficial effects on renal damage in addition to standard RAS blockade alone.

\section{Statistical Analyses}

Graph-Pad Prism software (Graph Pad Software, San Diego, CA, USA) was used for analyses. All values are expressed as mean \pm SEM. Statistical analysis was done by Student $t$ test, Wilcoxon's signed-rank test for unpaired data, or one-way analysis of variance when appropriate, analysis of variance for repeated measurements, followed by Student-NewmanKeuls test performed for the analysis within groups, when appropriate. Comparison of 


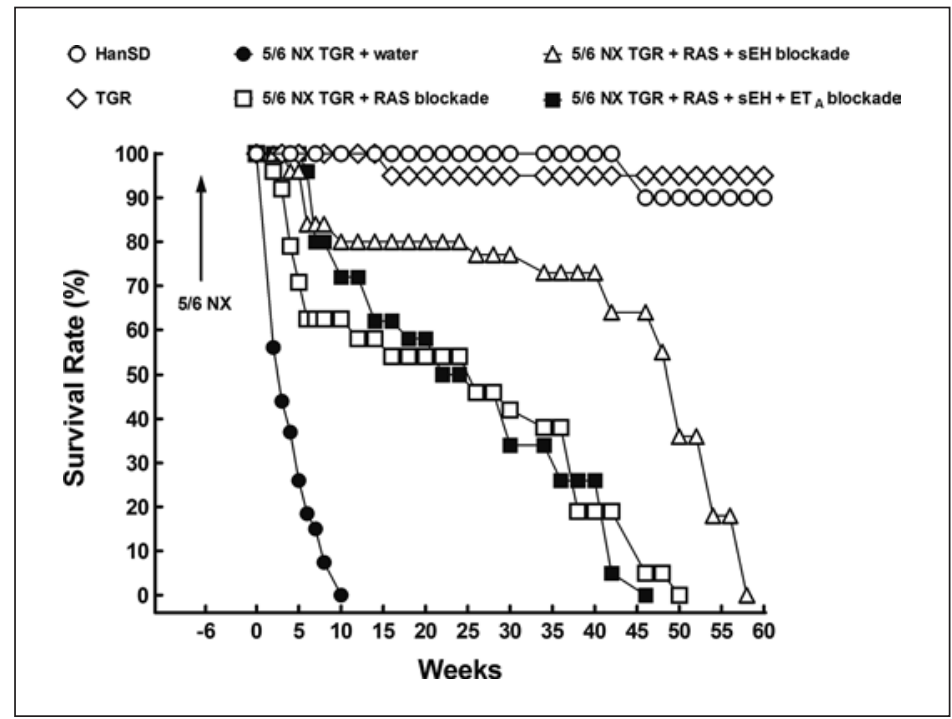

Fig. 1. Survival rate in sham-operated HanSD (transgene-negative) rats and in heterozygous Ren-2 TGRs, and in 5/6 nephrectomized (5/6 NX) TGR, untreated (water) or receiving RAS blockade (an angiotensin converting enzyme inhibitor plus an angiotensin II receptor blocker). Alternatively, the above 2-drug RAS blockade was combined with the sEH inhibitor. In addition, the complex RAS and sEH blockade was further combined with $\mathrm{ET}_{\mathrm{A}}$ receptor antagonist. 5/6 NX TGR + water group exhibited the worst survival rate among all experimental groups. 5/6 NX TGR + RAS + sEH blockade revealed better survival rate curve than 5/6 NX TGR + RAS blockade and 5/6 NX TGR + RAS + $\mathrm{SEH}+\mathrm{ET}_{\mathrm{A}}$ blockade (examined by log-rank Mantel-Cox test followed by Gehan-Breslow-Wilcoxon test). HanSD, Hannover-Sprague Dawley; TGR, transgenic rat; RAS, renin-angiotensin system; sEH, soluble epoxide hydrolase; $\mathrm{ET}_{\mathrm{A}}$, endothelin type A.

survival curves was performed by log-rank (Mantel-Cox) test followed by Gehan-BreslowWilcoxon test. In order to obtain reliable data regarding the effects of treatment regimens on the survival rate, high initial $n$ values were used (not so for sham-operated animals) to enable valid comparison of the long-term survival rate, and those initial $n$ values were estimated by statistical power analyses method developed by Cohen [36].

The values exceeding $95 \%$ probability limits $(p<0.05)$ were considered statistically significant.

\section{Results}

Series 1: The Effects of Treatments on Survival Rate, Albuminuria, and Clearance of Endogenous Creatinine

In both sham-operated TGR and HanSD group, one animal unexpectedly died during the experiment (for unknown reasons); however, the survival rate remained above $90 \%$ at the end of experiment. As shown in Figure 1, untreated 5/6 NX TGR began to die at week +1 (i.e., 7 weeks after 5/6 NX) and by week +10 no animal survived. The treatment with complex RAS blockade significantly improved the survival rate; however, beginning from week +10 , the survival rate considerably decreased throughout the study and all animals died by week +50 . Combined RAS and sEH blockade were more effective than complex RAS blockade alone, but survival decreased sharply after week +46 and no animal survived by week +58. Remarkably, combined complex RAS blockade together with $\mathrm{sEH}$ and $\mathrm{ET}_{\mathrm{A}}$ receptor blockade was not more effective than complex RAS blockade alone: throughout 
Fig. 2. Albuminuria (A) and creatinine clearance $(\mathbf{B})$ in sham-operated HanSD (transgene-negative) rats and in heterozygous Ren-2 TGRs, and in 5/6 nephrectomized (5/6 NX) TGR, untreated (water) or receiving RAS blockade (an angiotensin converting enzyme inhibitor plus angiotensin II receptor blocker). Alternatively, the above 2-drug RAS blockade was combined with the sEH inhibitor. In addition, the complex RAS and sEH blockade was also combined with $\mathrm{ET}_{\mathrm{A}}$ receptor antagonist. $* p<0.05$ compared with sham-operated HanSD rats at the same time point. ${ }^{\#} p<$ 0.05 compared with sham-operated TGR at the same time point. @ $p<0.05$ compared with all the other groups at the same time point. HanSD, Hannover-Sprague Dawley; TGR, transgenic rat; RAS, renin-angiotensin system; $\mathrm{sEH}$, soluble epoxide hydrolase; $\mathrm{ET}_{\mathrm{A}}$, endothelin type A.

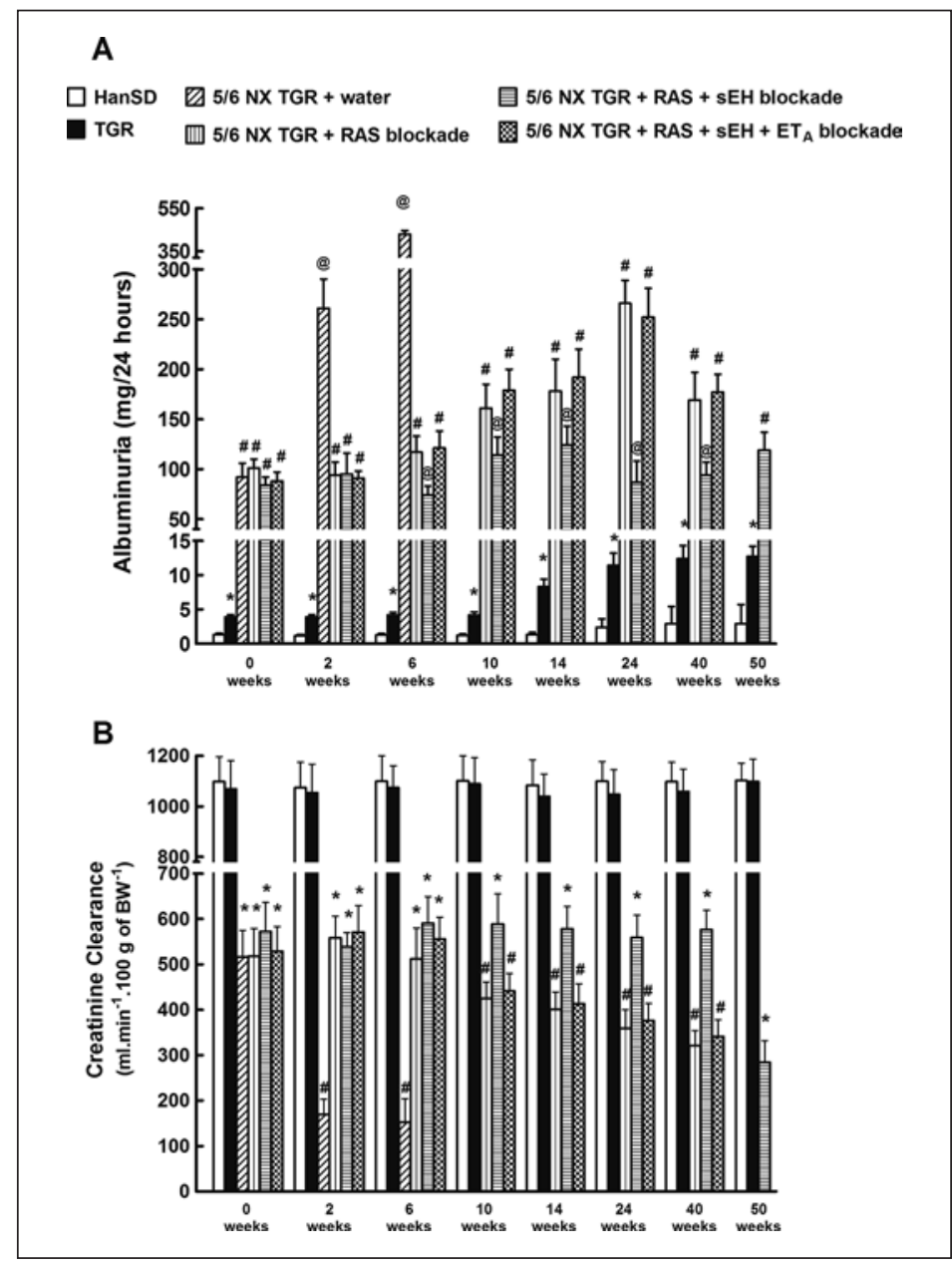

the experiment, there was no significant difference in the survival rate between these 2 treatment regimens (Fig. 1).

As shown in Figure 2A, sham-operated HanSD rats showed minimal albuminuria throughout the experiment (at the end: $2.9 \pm 2.8 \mathrm{mg} / 24 \mathrm{~h}$ ). Sham-operated TGR revealed significantly higher albuminuria than sham-operated HanSD rats (about 3-fold higher), which slightly but progressively increased throughout the experiment. At the end of study, it was about 4-fold higher than that observed in sham-operated HanSD rats. However, compared with animals after 5/6 NX, the albuminuria remained noticeably lower. Untreated 5/6 NX TGR before initiating of appropriate treatment regimen (on week 0 , i.e., 6 weeks after $5 / 6 \mathrm{NX}$ ) had albuminuria up to 20 -fold higher than in the sham-operated TGR, and on weeks +2 and +6 , it revealed a dramatic increase reaching the maximum on week $+6(469 \pm 48 \mathrm{mg} / 24 \mathrm{~h}$, $p<0.05$ vs. all other corresponding values). On weeks +2 and +6 , all treatment regimens were seen to attenuate albuminuria in 5/6 NX TGR, but it remained significantly higher than in sham-operated TGR. Remarkably, when viewed throughout the experiment, the combined complex RAS and sEH blockade was more effective in reducing albuminuria than either complex RAS blockade alone or the triad of complex RAS, $\mathrm{sEH}$, and $\mathrm{ET}_{\mathrm{A}}$ receptor blockade.

There were no significant differences in creatinine clearance between sham-operated TGR and HanSD rats throughout the study (Fig. 2B). Untreated 5/6 NX TGR demonstrated a profound decline in creatinine clearance on weeks +2 and +6 , which was also associated with 
Fig. 3. The ratio of albuminuria to creatinine clearance in sham-operated animals (A) and in 5/6 nephrectomized (5/6 NX) animals (B) in sham-operated HanSD (transgene-negative) rats and in heterozygous Ren-2 TGRs, untreated (water) or receiving RAS blockade (an angiotensin converting enzyme inhibitor and an angiotensin II receptor blocker). Alternatively, the above RAS blockade was combined with the sEH inhibitor. In addition, the RAS and sEH blockade was also combined with $\mathrm{ET}_{\mathrm{A}}$ receptor antagonist. $* p<0.05$ compared with sham-operated HanSD rats at the same time point. ${ }^{@} p<0.05$ compared with all the other unmarked groups at the same time point. HanSD, Hannover-Sprague Dawley; TGR, transgenic rat; RAS, renin-angiotensin system; sEH, soluble epoxide hydrolase; $\mathrm{ET}_{\mathrm{A}}$, endothelin type A.

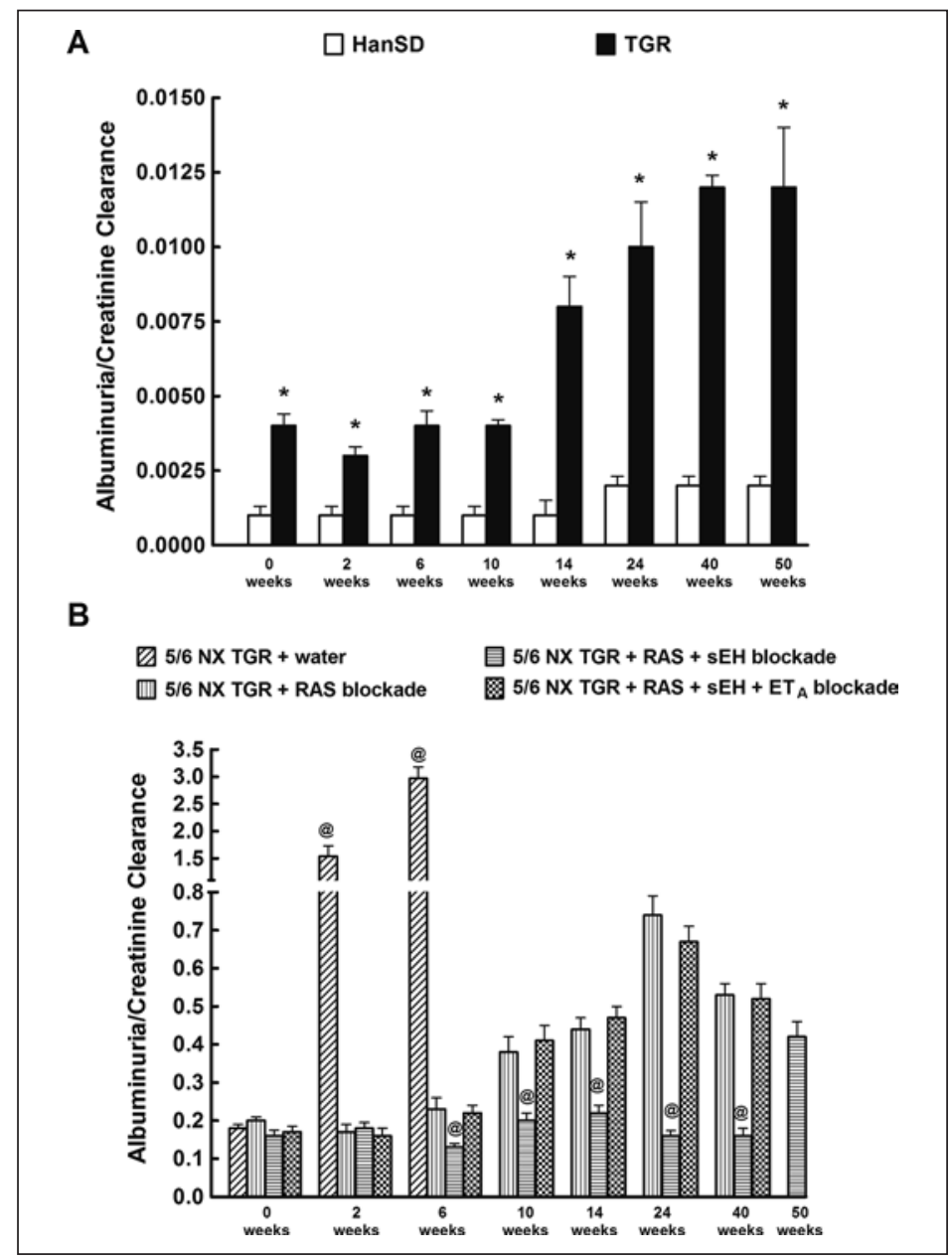

the dramatic onset of mortality (Fig. 1). All treatment regimens in 5/6 NX TGR attenuated these decreases; however, the creatinine clearance remained significantly lower than that observed in sham-operated TGR. Nevertheless, after week +10 , creatinine clearance in $5 / 6$ NX TGR treated either with complex RAS blockade alone or the triad of complex RAS blockade, $\mathrm{SEH}$ and $\mathrm{ET}_{\mathrm{A}}$ receptor blockade began to decline and was significantly lower that observed in 5/6 NX TGR treated with complex RAS plus sEH blockade.

As shown in Figure 3A, when albuminuria was normalized for glomerular filtration rate (the albuminuria-to-creatinine clearance ratio), it was minimal and stable in sham-operated HanSD rats throughout the study. On the other hand, in sham-operated TGR, it progressively increased and at the end of the study was about 6-fold higher than in the normotensive counterparts.

Using the same index, in untreated 5/6 NX TGR albuminuria on week +6 was 740 -fold higher (Fig. 3B) than observed in sham-operated TGR on the same week (Fig. 3A). As shown, all treatment regimens in 5/6 NX TGR reduced the albuminuria and, again, starting from week +6 , the complex RAS blockade plus sEH blockade was more effective than complex RAS blockade alone or the triad of combined complex RAS plus SEH inhibition and $\mathrm{ET}_{\mathrm{A}}$ receptor blockade. The 6-week treatment with the RAS blockade plus sEH blockade attenuated the albuminuria in 5/6 NX TGR more than 20-fold as compared with untreated $5 / 6$ NX TGR ( $0.13 \pm 0.01$ vs. $2.97 \pm 0.21, p<0.05$; Fig. $3 B$ ). Nevertheless, albuminuria on 
Fig. 4. Glomerulosclerosis index (GSI) (A) and kidney cortical tubulointerstitial injury (B) in shamoperated HanSD (transgene-negative) rats and in heterozygous Ren-2 TGRs, and in 5/6 nephrectomized (5/6 NX) TGR, untreated (water) or receiving RAS blockade (an angiotensin converting enzyme inhibitor and an angiotensin II receptor blocker). Alternatively, the above RAS blockade was combined with the sEH inhibitor. In addition, the RAS and sEH blockade was also combined with $\mathrm{ET}_{\mathrm{A}}$ receptor antagonist. $* p<0.05$ compared with sham-operated HanSD rats at the same time point. ${ }^{\#} p<0.05$ compared with all the other groups at the same time point. HanSD, Hannover-Sprague Dawley; TGR, transgenic rat; RAS, renin-angiotensin system; $\mathrm{sEH}$, soluble epoxide hydrolase; $\mathrm{ET}_{\mathrm{A}}$, endothelin type A.

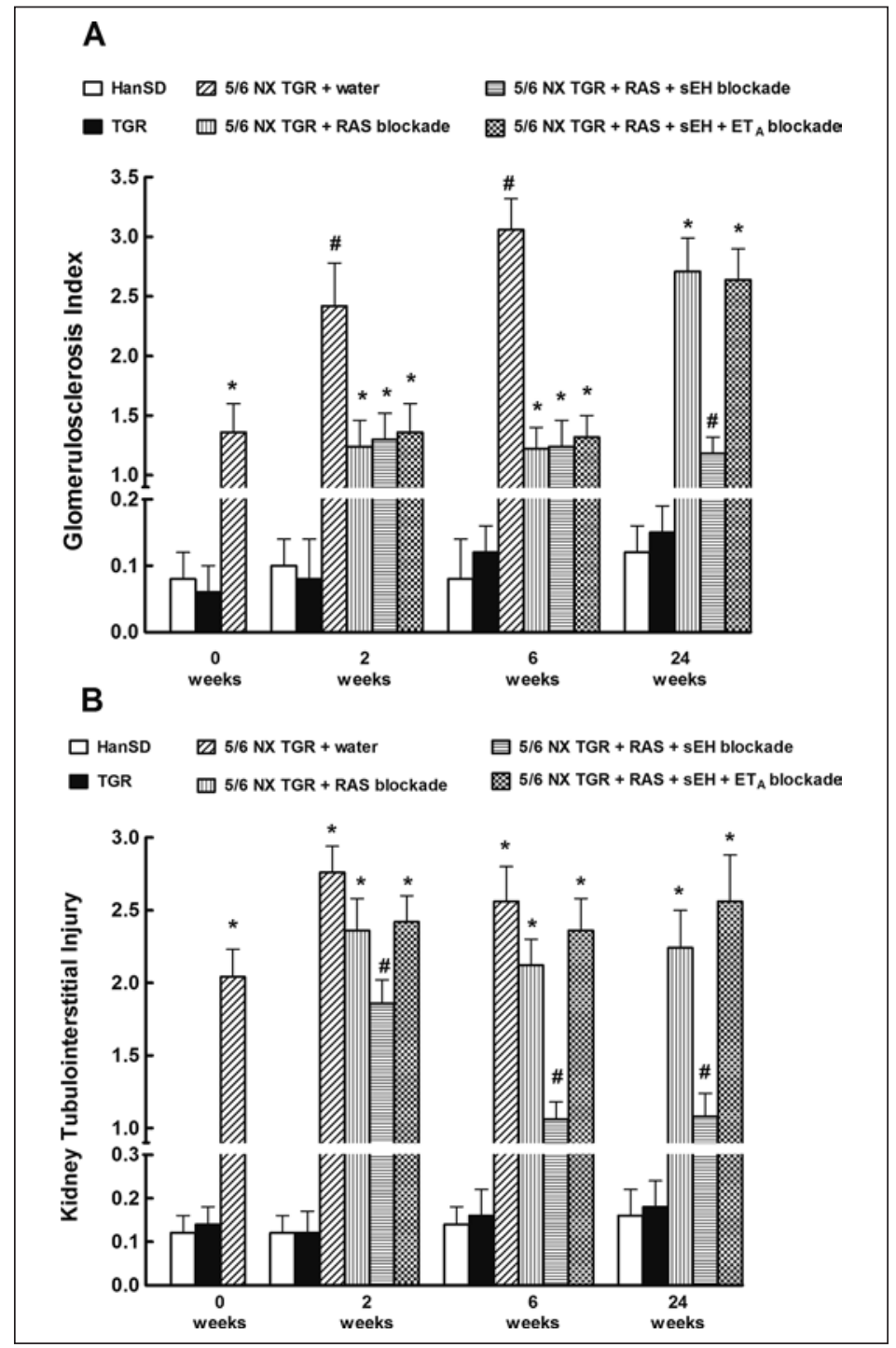

week +6 in 5/6 NX TGR treated with the RAS blockade and sEH blockade still remained about 30 -times higher than observed in sham-operated TGR $(0.13 \pm 0.01$ vs. $0.004 \pm 0.0005$, $p<0.05$; Fig. 3A, B).

Series 2: The Effects of Treatments on the Development of Renal Glomerular Damage and Renal Cortical Tubulointerstitial Injury

As shown in Figure 4A, already in week 0 (i.e., 6 weeks after 5/6 NX), untreated 5/6 NX TGR showed an obvious GSI increase, then the index increased further and in week +6 reached the value above 3 , indicating an enormously severe renal glomerular damage. Treatment with complex RAS blockade alone or combined complex RAS blockade and $\mathrm{sEH}$ inhibition plus $\mathrm{ET}_{\mathrm{A}}$ receptor blockade attenuated the increases in GSI in 5/6 NXTGR, but their effectiveness markedly decreased in the late phase (on week +24 , i.e., 30 weeks after $5 / 6 \mathrm{NX}$ ). In contrast, the treatment with complex RAS and sEH blockade remained effective throughout the experiment.

Figure 4B shows that kidney tubulointerstitial injury followed a change pattern analogous in part with that observed for GSI; however, the RAS blockade alone and the RAS plus $\mathrm{sEH}$ plus $\mathrm{ET}_{\mathrm{A}}$ triad treatment were virtually ineffective over the whole follow-up period. 


\section{Kidney \\ Blood Pressure \\ Research}

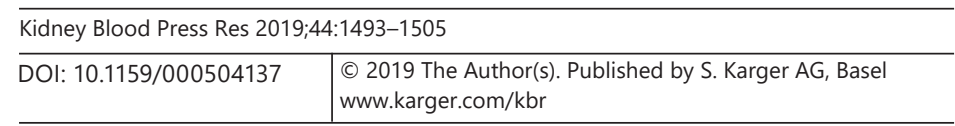

Čertíková Chábová et al.: RAS, sEH, and $\mathrm{ET}_{\mathrm{A}}$ Blockade in 5/6 Renal Ablation

\section{Discussion}

The crucial finding of the present study is that chronic treatment with sEH inhibitor when added to the complex RAS blockade in animals with established CKD brought additional renoprotective effects and attenuated the progression of CKD in 5/6 NX TGR. However, addition of $\mathrm{ET}_{\mathrm{A}}$ receptor blockade to this treatment regimen did not show additional benefit but, surprisingly, eliminated the beneficial effects of the combined RAS and sEH blockade. The present findings deserve special attention for several reasons.

First, a limited number of studies (including our earlier and the present ones) have evaluated treatment strategies in the phase of established CKD in the 5/6 NX model [28, 30, 37, 38]. It was clearly shown that when treatment was initiated in the established phase of CKD then the renoprotective effectiveness of the RAS blockade was significantly worse than when treatment was initiated shortly after $5 / 6 \mathrm{NX}$ induction $[3,4,15,16,22,24,29]$. These findings further support the notion that, in order to be relevant to the clinical situation, effectiveness of renoprotective strategies should be evaluated at a time when some renal damage is already present.

Our present results further support the evidence that renoprotection afforded by RAS blockade alone is not extended to the late stage of CKD. It was apparent that 5/6 NX TGR treated with complex RAS blockade showed decreasing survival rate and creatinine clearance, and increasing albuminuria, renal glomerular damage, and kidney tubulointerstitial injury. of special interest is the progressing increase in albuminuria because it is known that this parameter is correlated with a variety of important clinical outcomes, such as development of ESRD, cardiovascular disease, and all-cause mortality [5, 39]. Our results show, that even if the complex RAS blockade attenuated the albuminuria, it remained, when normalized to glomerular filtration rate, noticeably higher than in sham-operated TGR.

Special attention is also due to the additional renoprotective effects of the combined RAS and sEH blockade in 5/6 NX TGR as compared with the complex RAS blockade alone: not only improved survival rate but also further attenuation of albuminuria was seen. However, even though attenuated, the albuminuria in 5/6 NX TGR treated with the complex RAS and sEH blockade still remained elevated: in the critical point of a profound decrease in survival rate (i.e., in week +40), it was 13 fold higher than in sham-operated TGR and 80 fold higher than in sham-operated HanSD rats. Nevertheless, it was still $>4$ fold lower than in 5/6 NX TGR treated with the complex RAS blockade alone. In addition, it is important to acknowledge that by week +40 the survival rate in 5/6 NX TGR treated with the complex RAS and sEH blockade decreased to $73 \%$ and in 5/6 NX TGR treated with the complex RAS blockade alone to $38 \%$. It is apparent that between weeks +24 and +40 the animals with the highest albuminuria and lowest creatinine clearance were dying, which causes underestimation of the impact on albuminuria (a paradox of dying-dependent alteration of the estimate of albuminuria).

Why was it that addition of $\mathrm{ET}_{\mathrm{A}}$ receptor blockade to the combined RAS and $\mathrm{SEH}$ blockade not only did not bring any additional benefits but even abolished the beneficial renoprotective actions of the combined RAS plus sEH inhibition?

As evident from our recent [28] and the present findings, even if the dual RAS and sEH blockade exhibited the best renoprotective actions observed so far [18, 19, 22, 24, 27, 30, 31], there is still considerable space for the therapeutic improvement. As is obvious from the actual course of survival rate and proteinuria (particularly in the late phase of experiment), the treated animals fared distinctly worse than did the sham-operated ones. Therefore, our attention focused on the ET system which we previously showed to be markedly activated after renal mass reduction in TGR $[19,24]$. This activation augmented as CKD progressed toward the advanced CKD stage [30]. It will be noticed here that intrarenal overproduction 


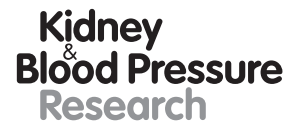

Kidney
Blood Pressure

Research

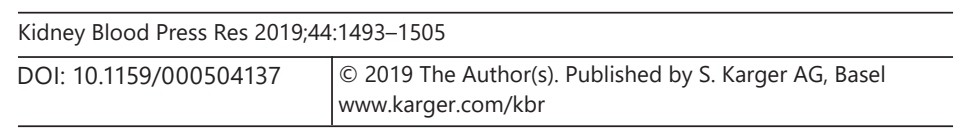

Čertíková Chábová et al.: RAS, sEH, and $\mathrm{ET}_{\mathrm{A}}$ Blockade in 5/6 Renal Ablation

of $\mathrm{ET}-1$ and $\mathrm{ET}_{\mathrm{A}}$ receptor activation follows the same pathways that lead to the renal fibrosis and glomerulosclerosis induced by angiotensin II [11, 12, 21]. Moreover, important stimulatory interactions, at various levels, were found between the RAS and ET systems [12, 22, 40, 41]. Furthermore, pharmacological blockade of $\mathrm{ET}_{\mathrm{A}}$ receptors either alone or added to the complex blockade of the RAS brought important renoprotective effects in 5/6 NX TGR, at least when the treatment was introduced immediately after the renal mass reduction, that is, before any substantial kidney injury developed ("early treatment protocol") [19, 22, 24]. In the light of these facts, a sound rationale has been provided for addition of $\mathrm{ET}_{\mathrm{A}}$ receptor blockade to the combined RAS and sEH blockade in an attempt to treat CKD progression, particularly in the advanced phase of the disease.

The failure of $\mathrm{ET}_{\mathrm{A}}$ receptor blockade to show renoprotective effects additional to those induced by the combined RAS and sEH blockade was unexpected. However, even more astonishing was our finding that such addition eliminated the beneficial effects of sEH blockade (when added to RAS blockade) and virtually returned the treatment effectiveness to the level observed with RAS blockade alone. We cannot provide a satisfactory explanation for this finding; however, we must recall here the results of our parallel study which showed no additional renoprotective actions of $\mathrm{ET}_{\mathrm{A}}$ receptor blockade when added to complex RAS blockade (a dual treatment regimen and not the triad applied now) in the advanced phase of CKD in 5/6 NX TGR [30]. Taking into consideration the results of both our studies, we propose that in the very advanced phase of CKD, at least in 5/6 NX TGR, apart from the RAS and ET systems and CYP-dependent metabolites of arachidonic acid other neurohormonal systems must be involved. Moreover, the divergence of the results of the same pharmacological regimen when applied as "early treatment" versus "late treatment protocol" further exposes the need for novel renoprotective treatment strategies to be evaluated in preclinical studies of advanced CKD. Apparently, such studies are more relevant to the clinical situations and could possibly be translated into clinical practice.

Nevertheless, it still remains mysterious why addition of $\mathrm{ET}_{\mathrm{A}}$ receptor blockade (application of a therapeutic triad) abolished the beneficial renoprotective actions of the treatment by combined complex RAS and sEH blockade in 5/6 NX TGR. In this connection, it is important to emphasize that there is a growing evidence that, at least in 5/6 NX rats, renoprotection is blood pressure (BP) dependent $[31,42]$. Since each of the 3 drugs employed in this study given alone exhibits significant BP-lowering action via different mechanisms [20, 25, 26, 34] in theory the combined therapy could have additive BP-lowering effect. If so, combined complex RAS blockade plus $\mathrm{sEH}$ inhibition plus $\mathrm{ET}_{\mathrm{A}}$ receptor blockade could bring BP below the renal blood flow autoregulation range $[43,44]$. This might per se impair renal function and thereby oppose the renoprotective effects of the treatment. However, data from our recent studies $[28,30]$ and preliminary experiments in which BP was monitored by radiotelemetry in 5/6 NX TGR starting 6 weeks after renal mass reduction for 3 weeks show that within $72 \mathrm{~h}$ after initiation of the complex RAS blockade BP decreased to levels that were not significantly different from the values measured in sham-operated HanSD rats. Admittedly, we have no data on the long-term effect of the treatment on BP. All the same, it seems that addition of either $\mathrm{SEH}$ inhibitor or $\mathrm{ET}_{\mathrm{A}}$ receptor antagonist or their combination would not bring any additional BP-lowering effect. Therefore, one can assume that exaggerated BP-lowering effect was not the reason for the elimination of the beneficial effects of the combined RAS and sEH blockade in 5/6 NX TGR.

It is important to recognize that our present study reveals some limitations; for example, the evaluation of indices of kidney injury employed in our present study differs from the methods that are used in the clinical nephrology. However, it is important to emphasize that the method employed in our experimental study was introduced by Nakano et al. [35], which is currently accepted as a gold standard for experimental studies in nephrology and, moreover, 
all our previous studies employed this semi-quantitative scale. Therefore, in order to compare the results of our previous (Vaněčková et al. [19]; Čertíková Chábová et al. [24]), current (Sedláková et al. [30]; Čertíková Chábová et al. [28]), and ongoing studies, we preserved this scoring method for the evaluation of kidney injury.

\section{Conclusion}

This study further strengthens the evidence that addition of pharmacological inhibition of sEH to the complex RAS blockade brings additional renoprotective effects and retards the progression of CKD in 5/6 NX TGR, also when the treatment is started in the established phase of CKD. The present data also show that addition of $\mathrm{ET}_{\mathrm{A}}$ receptor blockade to this treatment regimen does not bring additional renoprotection, in contrast, it was found to abolish the evident beneficial effects of the combined complex RAS and sEH blockade. All this information derived from the present experiments should be considered in attempts to develop new pharmacological strategies aimed at slowing down the progression of CKD to ESRD. So far, targeting CYP-derived metabolites of arachidonic acid at the level of sEH combined with RAS blockade seems a very promising approach.

\section{Statement of Ethics}

The studies followed the guidelines and practices established by the Animal Care and Use Committee of the Institute for Clinical and Experimental Medicine, which accord with the national law and with American Physiological Society guiding principles for the care and use of vertebrate animals in research and training, and was approved by this Committee and consequently by the Ministry of Health of the Czech Republic (the decision number for this project was 31102/2014-0ZV-30.0.3.6.14).

\section{Disclosure Statement}

The authors have no conflicts of interest to declare.

\section{Funding Sources}

This study was primarily supported by the Ministry of Health of the Czech Republic grants no. 15-28671A to V.Č.C. All rights reserved. This work was also supported by the National Institute of Diabetes and Digestive and Kidney Diseases DK103616 to J.I.

\section{Author Contribution}

V.Č.C., J.S., E.K.-J., V.T, J.I., I.V., and L.Č. primarily conceived and designed the study, analyzed and interpreted the data, and wrote the manuscript. P.K. and H.M. performed histological examination. V.Č.C., P.Š., I.V., and H.M. performed renal mass reduction. Z.V. performed biochemical analyses. B.H. designed and synthesized the sEH inhibitor. All authors were involved in the final analysis and interpretation of the data and contributed to the intellectual content and editing of the manuscript and approved its final version. 


\section{References}

1 Jha V, Garcia-Garcia G, Iseki K, Li Z, Naicker S, Plattner B, et al. Chronic kidney disease: global dimension and perspectives. Lancet. 2013 Jul;382(9888):260-72.

2 U.S. Renal Data System: USRDS. Annual Data Report 2015. Epidemiology of kidney disease in the United States.

3 Brenner BM. Nephron adaptation to renal injury or ablation. Am J Physiol. 1985 Sep;249(3 Pt 2):F324-37.

4 Zoja C, Abbate M, Remuzzi G. Progression of chronic kidney disease: insights from animal models. Curr Opin Nephrol Hypertens. 2006 May;15(3):250-7.

5 Zhong J, Yang HC, Fogo AB. A perspective on chronic kidney disease progression. Am J Physiol Renal Physiol. 2017 Mar;312(3):F375-84.

6 Raij L. The pathophysiologic basis for blocking the renin-angiotensin system in hypertensive patients with renal disease. Am J Hypertens. 2005 Apr;18(4 Pt 2):95S-9S.

7 Wheeler DC, Becker GJ. Summary of KDIGO guideline. What do we really know about management of blood pressure in patients with chronic kidney disease? Kidney Int. 2013 Mar;83(3):377-83.

8 Der Mesropian PJ, Shaikh G, Cordero Torres E, Bilal A, Mathew RO. Antihypertensive therapy in nondiabetic chronic kidney disease: a review and update. J Am Soc Hypertens. 2018 Mar;12(3):154-81.

9 Turner JM, Bauer C, Abramowitz MK, Melamed ML, Hostetter TH. Treatment of chronic kidney disease. Kidney Int. 2012 Feb;81(4):351-62.

10 Breyer MD, Susztak K. Developing treatments for chronic kidney disease in the 21st century. Semin Nephrol. 2016 Nov; 36(6):436-47.

11 Czopek A, Moorhouse R, Webb DJ, Dhaun N. Therapeutic potential of endothelin receptor antagonism in kidney disease. Am J Physiol Regul Integr Comp Physiol. 2016 Mar;310(5):R388-97.

12 Komers R, Plotkin H. Dual inhibition of renin-angiotensin-aldosterone system and endothelin-1 in treatment of chronic kidney disease. Am J Physiol Regul Integr Comp Physiol. 2016 May;310(10):R877-84.

13 Morrison AB. Experimentally induced chronic renal insufficiency in the rat. Lab Invest. 1962 Apr;11:321-32.

14 Shimamura T, Morrison AB. A progressive glomerulosclerosis occurring in partial five-sixths nephrectomized rats. Am J Pathol. 1975 Apr; 79(1):95-106.

15 Hostetter TH. Hyperfiltration and glomerulosclerosis. Semin Nephrol. 2003 Mar;23(2):194-9.

16 Čertíková Chábová V, Červenka L. The dilemma of dual renin-angiotensin system blockade in chronic kidney disease: why beneficial in animal experiments but not in the clinic? Physiol Res. 2017 May;66(2): 181-92.

17 Yanagisawa M, Kurihara H, Kimura S, Tomobe Y, Kobayashi M, Mitsui Y, et al. A novel potent vasoconstrictor peptide produced by vascular endothelial cells. Nature. 1988 Mar;332(6163):411-5.

18 Vanêcková I, Kramer HJ, Bäcker A, Vernerová Z, Opočenský M, Červenka L. Early endothelin-A receptor blockade decreases blood pressure and ameliorates end-organ damage in homozygous Ren-2 rats. Hypertension. 2005 Oct; 46(4):969-74.

19 Vaněčková I, Kujal P, Husková Z, Vaňourková Z, Vernerová Z, Certíková Chábová V, et al. Effects of combined endothelin A receptor and renin-angiotensin system blockade on the course of end-organ damage in 5/6 nephrectomized Ren-2 hypertensive rats. Kidney Blood Press Res. 2012;35(5):382-92.

20 Davenport AP, Hyndman KA, Dhaun N, Southan C, Kohan DE, Pollock JS, et al. Endothelin. Pharmacol Rev. 2016 Apr;68(2):357-418.

21 De Miguel C, Speed JS, Kasztan M, Gohar EY, Pollock DM. Endothelin-1 and the kidney: new perspectives and recent findings. Curr Opin Nephrol Hypertens. 2016 Jan;25(1):35-41.

22 Vaněčková I, Hojná S, Kadlecová M, Vernerová Z, Kopkan L, Červenka L, et al. Renoprotective effects of ET(A) receptor antagonists therapy in experimental non-diabetic chronic kidney disease: is there still hope for the future? Physiol Res. 2018 Jun;67 Suppl 1:S55-67.

23 Mullins JJ, Peters J, Ganten D. Fulminant hypertension in transgenic rats harbouring the mouse Ren-2 gene. Nature. 1990 Apr;344(6266):541-4.

24 Čertíková Chábová V, Vernerová Z, Kujal P, Husková Z, Škaroupková P, Tesař V, et al. Addition of ET(A) receptor blockade increases renoprotection provided by renin-angiotensin system blockade in 5/6 nephrectomized Ren-2 transgenic rats. Life Sci. 2014 Nov;118(2):297-305.

25 Fan F, Roman RJ. Effect of cytochrome P450 metabolites of arachidonic acid in nephrology. J Am Soc Nephrol. 2017 Oct;28(10):2845-55.

26 Imig JD. Prospective for cytochrome P450 epoxygenase cardiovascular and renal therapeutics. Pharmacol Ther. 2018 Dec;192:1-19.

27 Kujal P, Čertíková Chábová V, Škaroupková P, Husková Z, Vernerová Z, Kramer HJ, et al. Inhibition of soluble epoxide hydrolase is renoprotective in 5/6 nephrectomized Ren-2 transgenic hypertensive rats. Clin Exp Pharmacol Physiol. 2014 Mar; 41(3):227-37.

28 Čertíková Chábová V, Kujal P, Škaroupková P, Varňourková Z, Vacková Š, Husková Z, et al. Combined inhibition of soluble epoxide hydrolase and renin-angiotensin system exhibits superior renoprotection to renin-angiotensin system blockade in 5/6 nephrectomized Ren-2 transgenic hypertensive rats with established chronic kidney disease. Kidney Blood Press Res. 2018;43(2):329-49.

29 Sangalli F, Carrara F, Gaspari F, Corna D, Zoja C, Botti L, et al. Effect of ACE inhibition on glomerular permselectivity and tubular albumin concentration in the renal ablation model. Am J Physiol Renal Physiol. 2011 Jun; 300(6):F1291-300. 
30 Sedláková L, Čertíková Chábová V, Doleželová Š, Škaroupková P, Kopkan L, Husková Z, et al. Renin-angiotensin system blockade alone or combined with ETA receptor blockade: effects on the course of chronic kidney disease in 5/6 nephrectomized Ren-2 transgenic hypertensive rats. Clin Exp Hypertens. 2017;39(2):183-95.

31 Kujal P, Chábová VČ, Vernerová Z, Walkowska A, Kompanowska-Jezierska E, Sadowski J, et al. Similar renoprotection after renin-angiotensin-dependent and -independent antihypertensive therapy in 5/6-nephrectomized Ren-2 transgenic rats: are there blood pressure-independent effects? Clin Exp Pharmacol Physiol. 2010 Dec;37(12):1159-69.

32 Fujihara CK, Velho M, Malheiros DM, Zatz R. An extremely high dose of losartan affords superior renoprotection in the remnant model. Kidney Int. 2005 May;67(5):1913-24.

33 Sporková A, Jíchová S, Husková Z, Kopkan L, Nishiyama A, Hwang SH, et al. Different mechanisms of acute versus long-term antihypertensive effects of soluble epoxide hydrolase inhibition: studies in Cyp1a1-Ren-2 transgenic rats. Clin Exp Pharmacol Physiol. 2014 Dec;41(12):1003-13.

34 Vaněčková I, Dobešová Z, Kuneš J, Vernerová Z, Zicha J. Endothelin A receptor blocker atrasentan lowers blood pressure by the reduction of nifedipine-sensitive calcium influx in Ren-2 transgenic rats fed a high-salt diet. J Hypertens. 2015 Jan;33(1):161-9.

35 Nakano Y, Hirano T, Uehara K, Nishibayashi S, Hattori K, Aihara M, et al. New rat model induced by antiglomerular basement membrane antibody shows severe glomerular adhesion in early stage and quickly progresses to end-stage renal failure. Pathol Int. 2008 Jun;58(6):361-70.

36 Cohen J. Statistical Power Analysis for the Behavioral Sciences. Academic press; 2013. pp. 1-17.

37 Arias SC, Valente CP, Machado FG, Fanelli C, Origassa CS, de Brito T, et al. Regression of albuminuria and hypertension and arrest of severe renal injury by a losartan-hydrochlorothiazide association in a model of very advanced nephropathy. PLoS One. 2013;8(2):e56215.

38 Arias SC, Souza RA, Malheiros DM, Fanelli C, Fujihara CK, Zatz R. An association of losartan-hydrochlorothiazide, but not losartan-furosemide, completely arrests progressive injury in the remnant kidney. Am J Physiol Renal Physiol. 2016 Jan;310(2):F135-43.

39 Inker LA, Astor BC, Fox CH, Isakova T, Lash JP, Peralta CA, et al. KDOQI US commentary on the 2012 KDIGO clinical practice guideline for the evaluation and management of CKD. Am J Kidney Dis. 2014 May;63(5):71335.

40 Barton M, Shaw S, d'Uscio LV, Moreau P, Lüscher TF. Angiotensin II increases vascular and renal endothelin-1 and functional endothelin converting enzyme activity in vivo: role of ETA receptors for endothelin regulation. Biochem Biophys Res Commun. 1997 Sep;238(3):861-5.

41 Rossi GP, Sacchetto A, Cesari M, Pessina AC. Interactions between endothelin-1 and the renin-angiotensinaldosterone system. Cardiovasc Res. 1999 Aug;43(2):300-7.

42 Bidani AK, Polichnowski AJ, Loutzenhiser R, Griffin KA. Renal microvascular dysfunction, hypertension and CKD progression. Curr Opin Nephrol Hypertens. 2013 Jan;22(1):1-9.

43 Navar LG. Renal autoregulation: perspectives from whole kidney and single nephron studies. Am J Physiol. 1978 May;234(5):F357-70.

44 Carlström M, Wilcox CS, Arendshorst WJ. Renal autoregulation in health and disease. Physiol Rev. 2015 Apr; 95(2):405-511. 